\title{
Verzeichnis von öfter abgekürzt angeführten Werken.
}

Die in [] angeführten Schriften sind nach AbschluB des Werkes erschienen.

A less andri, Rodriguez, Arturo, De la compra-venta i de la promesa de venta. T. 1. 2. Santiago [Chile] 1917-18.

A i má s i, Anton, Ungarisches Privatrecht. Bd. 1. 2. Berlin \& Leipzig 1923-24. (Ungarische Bibliothek. Reihe 2, Nr. 1. 3.)

Almé n: Almén, Tore, Das skandinavische Kaufrecht. Deutsche Ausg. von Friedrich Karl Ne ubecker. Bd. 1-3. Heidelberg 1922.

Almé n': Almén, Tore, Om köp och byte av lös egendom. Kommentar till lagen den 20 Juni 1905. 3, delvis omarb. uppl. av Rudolf E k l u n d. D. 1. 2. Stockholm 1934.

A $1 \mathrm{mé}$ n, Lagen om avtal: Almén, Tore, och Rudolf Ek l u nd, Lagen om avtal och andra rättshandlingar på förmögenhetsrättens område av den 11 Juni 1915. 3. uppl. Stockholm 1931.

A u b r y e t $\mathrm{R}$ a u: Cours de droit civil français d'après la méthode de Zachariae. 5. éd. par G. Rau, Ch. Falcinnaire, M. Gault. T. 1-12. Paris 1897-1922.

B a r a s i, L., Istituzioni di diritto civile. 2. ed. Milano 1921. (Biblioteca giuridica contemporanea.)

B a r t $\mathrm{sch}$, Robert, und Rudolf Pollak, Konkurs-, Ausgleichs-, Anfechtungsordnung und deren Einführungsverordnung. 2. Aufl. Wien \& Leipzig 1927. [3. neubearb. Aufl. seit 1935 im Erscheinen.]

Ba udry-Lacantinerie, G., Traité théorique et pratique de droit civil. 12-15. - et L. B a r d e, Des obligations. 3. éd. T. 1-4. Paris 1906.

19. - et Léo $\mathrm{S}$ a igna $\mathrm{t}$, De la vente et de l'échange. 3. éd. Paris 1908. Supplément par Julien Bonneça s e. T. 1-6. Paris 1924-1935.

B e cke r, H., Obligationenrecht. Abt. 1. 2. Bern 1917-23. (Kommentar zum schweizerischen Zivilgesetzbuch hrsg. von $M$. Gmür. Bd. 6.)

B e $\mathrm{n}$ j a m in, Judah Philip, A Treatise on the law of sale of personal property with references to the French code and civil law. 7 . ed. by A. R. Ke nn e d y. London 1931.

B e $n$ it o, Lorenzo, Manual de derecho mercantil. 3. ed. T. 1. 2.; 1. ed. T. 3. Madrid 1924-29.

B e n to de Faria, Antonio, Código commercial brasileiro. Annotado. 1. 2. Rio de Janeiro 1920

Blanco Constans, Francisco, Estudios elementales de derecho mercantil. 3. ed., notabl. corr. y aum. T. 1. 2. Madrid 1910-11.

B o l a f $\mathrm{i}$ o, Leone, Leggi ed usi commerciali. Atti di commercio. Dei commercianti. Dei libri di commercio. (Art. 1 a 28 cod. comm.) 6. ed. Torino 1935. (Il Codice di Commercio commentato. Vol. 1.)

Bonelli, Gustavo, Del fallimento (commento al codice di commercio). Vol. 1-3. Milano 1923. (Biblioteca giuridica contemporanea.)

d e B o o r, H. O., Die Kollision von Forderungsrechten. Berlin 1928.

Brenes Córdoba, Alberto, Tratado de las obligaciones y contratos. San José 1923. (Derecho civil de Costa Rica.)

B r u net t i, Antonio, Diritto fallimentare italiano. Roma 1932.

C a n di a n, Aurelio, Il processo di fallimento. Programma di un corso. Padova 1934.

C a n s te i n, R. Frhr. von, Lehrbuch des österreichischen Handelsrechtes, unter steter Berücks. der Rechtsprechung des Obersten Gerichtshofes. Bd. 1. 2. Berlin 1895-96. 
Capitant, Henri, De la Cause des obligations (contrats, engagements unilatéraux, legs). 3. éd. Paris 1927.

Carvalho de Mendonça, José Xavier, Tratado de direito commercial brasileiro. 2. ed. Vol. 1-7. Rio de Janeiro 1933-34.

Cassin, René, De l'Exception tirée de' l'inexécution dans les rapports synallagmatiques (exceptio non adimpleti contractus). Paris 1914. Thèse.

Cha l mers' (M. D.) Sale of Goods Act, 1893, including the factors acts, 1889 \& 1890. 11. ed. by Ralph Sutt on and N. P. Sh a n n o n. London 1931.

Ch i r oni, G. P., La colpa nel diritto civile odierno. 2. ed. interam. rif. (Ristampa). Colpa contrattuale. Torino 1925. (Nuova collezione di opere giuridiche. No. 37.)

Chitty's Treatise on the law of contracts. 18. ed. by W. A. MacFarlane and $\mathrm{G}$. W. Wr a $\mathrm{ng}$ h a $\mathrm{m}$. London 1930.

Colin, Ambroise, et $H$. C a p it a $\mathrm{t}$, Cours élémentaire de droit civil français. 7. éd. T. 1-3 [nebst] suppl. Paris 1931-34. [8. éd. T. 1. 2. 1934-35.]

Coll i er, W. M. Miller, The Law and practice in bankruptcy under the national bankruptcy act of 1898 official and supplemental forms. 13. ed. by Frank B. Gilbert and Fred E. Ros brook. Vol. 1-4. [nebst] suppl. Albany $1923-35$.

Cortes, Felix, Comentarios al código de comercio terrestre. Bogotá 1933.

Cunha Gonçalves (I-IX): Da Cunha Gonçalves, Luis, Tratado de direito civil em comentário ao código civil português, Vol. 1-9. Coimbra 1929-34.

Cunha Goncalves²: Da Cunha Gonçalves, Luis, Da compra e venda no direito commercial português. 2. ed. Coimbra 1924.

Cunha Gonçalves: Da Cunha Gonçalves, Luis, Da compra e venda no direito commercial brasileiro. S. Paulo 1924.

Cut u ri, Torquato, Della vendita, della cessione e della permuta. Ristampa stereot. d. 2. ed. riv. ed. aum. Napoli 1923. (Il diritto civile italiano secondo la dottrina e la giurisprudenza. P. 12.)

Dalloz, Rép. prat.: Dalloz, Répertoire pratique de législation, de doctrine et de jurisprudence publ. sous la dir. de Gaston Griolet et Charles Vergé. T. 1-12 [nebst] suppl. T. 1-3. Paris 1910-31.

D e l a y e n, G., R. H o m b u r g et Gaston $\mathrm{Ch}$ o $\mathrm{t}$ i a $\mathrm{u}$, Des marchés commerciaux. Paris 1927.

Dem og u e, René, Traité des obligations en général. T. 1-7. Paris 1923-33.

Demolombe, C., Traité des contrats ou des obligations conventionnelles en général. T. 1-8. Paris 1877-1882. (Cours de cođe Napoléon. 24-31.)

$\mathrm{D}$ ü $\mathrm{r}$ in $\mathrm{g}$ e $\mathrm{r}-\mathrm{H}$ a c h e n b u r g, Das Handelsgesetzbuch vom 10. Mai 1897 (unter Ausschluß des Seerechts) auf der Grundlage des Bürgerlichen Gesetzbuchs. 3. Aufl, Bd. 1-5. Mannheim, Berlin, Leipzig 1930-32.

E c há v a r r i y Vivanco, José M. G., Comentarios al código de comercio. 2. ed. T. 1-5. Valladolid 1928.

Ehre nz we i g, Armin, System des österreichischen allgemeinen Privatrechts. 6. Aufl. Bd. 1. 2, 2; 7. Aufl. Bd. 2, 1. Wien 1923-28.

Enneccerus-Lehm a n n: Enneccerus, Ludwig, Lehrbuch des bürgerlichen Rechts. Bd. 2. Recht der Schuldverhältnisse, 12. Bearb. von Heinrich Lehmann. Marburg 1932.

Es me in s. Planiol et Ripert.

Fest s ch rif t f ü r B e k ker: Aus römischem und bürgerlichem Recht, Ernst Immanuel Bekker zum 16. August 1907 überreicht von F. Bernhöft, P. F. Girard [u. a.]. Weimar 1907.

F i ck-S ch n e i der, OR.: Das schweizerische Obligationenrecht vom 30. März 1911. 1. Aufl. zugleich 4. Aufl. d. Kommentars von A. Schneider u. H. Fick. Titel 1-22 hrsg. von F. Fick, 1911. Titel 23 - Schluß hrsg. von G. Bachmann, F. Goetzinger [u. a.] 1915. [nebst] alph. Sachregister 1916. Zürich.

Fischer-Hen1e-Titze: Bürgerliches Gesetzbuch. Handausg. von Otto Fischer u. Wilhelm Henle. 14. Aufl. hrsg. von Heinrich Titze. München 1932. 
Frédéricq, Louis, Principes de droit commercial belge. T. 1-3. Gand $1928-34$.

Gasca, Cesare Luigi, Trattato della compra-vendita civile e commerciale, 2. ed. Vol. 1. 2. Torino 1914-15.

Ga up -Ste in-J on a s: Gaupp, Ludwig, und Friedrich Stein, Kommentar zur Zivilprozeßordnung in der Fassung der Bekanntmachung vom 8. November 1933. 15. neubearb. Aufl. von Martin Jonas. Bd. 1. 2. Tübingen 1934.

Gay de Montella, R., Tratado de la legislación comercial española a base del código de comercio. T. $1-6$. Barcelona 1930.

Giorgi, Giorgio, Teoria delle obbligazioni nel diritto moderno italiano. Vol. 1-9. 7, ed. Firenze 1910-26.

Gloag, William Murray, The Law of contract. A treatise on the principles of contract in the law of Scotland. 2. ed. Edinburgh 1929.

Gloag and Henderson: Gloag. William Murray, and Robert Candlish Henderson, Introduction to the law of Scotland. 2. ed. Edinburgh 1933.

G o i t e in s. Smith-Gutteridge.

G o u d o e ver, H. van, Verbintenissenrecht, Voortgezet door J. Limburg. 2. dr. stuk 1. Zwolle o. J. (Asser, C., Handleiding tot de beoefening van het Nederlandsch burgerlijk recht. D. 3.)

Gro B m a n n-D o e rth, Hans, Das Recht des Uberseekaufs. Bd. 1. Mannheim, Berlin, Leipzig 1930. (Uberseestudien $z$. Handels-, Schiffahrts- und Versicherungsrecht. $H$. 11.)

GroBmann-Doerth, Hans, Die Rechtsfolgen vertragswidriger Andienung. Marburg 1934. (Arbeiten z. Handels-, Gewerbe- u. Landwirtschaftsrecht. Nr. 74.)

Grundtvig, L. A., Lov om Kab af 6. April 1906. 2. rev. Udg. ved Alf Ross. København 1922.

Guhl, Theo, Das schweizerische Obligationenrecht mit EinschluB des Handels-, Wechsel- und Versicherungsvertragsrechts. Zürich 1933.

Gu i ma rães, Aureliano, A compra e venda civil. S. Paulo 1927.

H a g e r u p. Prancis, Konkurs og Akkordforhandling. 4. Udg. ved. P. J. Paulsen. Oslo 1932 .

$\mathrm{H}$ a h n, Friedrich von, Kommentar zum Allgemeinen Deutschen Handelsgesetzbuch. 2.-3. Aufl, Bd. 1. 2. Braunschweig 1875-77.

Hallager-A ubert: Hallager, Fr., Den norske Obligationsrets almindelige Del. Omarb. og forgget Udg. af nogle Afsnit af ,Den-norske Obligationsret v. L. M. B. Aubert. 2. uforandr. Opl. Christiania 1896.

$\mathrm{H}$ als bury's Laws of England being a complete statement of the whole law of England. 2. ed. under the general editorship of $\mathrm{Hailsham}$. Vol. 7. London 1932.

$\mathrm{H}$ a $\mathrm{n} \mathrm{d} \mathrm{b} \mathrm{u} \mathrm{ch}$ des Landesproduktenhandels 1929. Mit dem ... Kommentar zu den Einheitsbedingungen im deutschen Getreidehandel. 2. neubearb. u. erw. Ausg. Berlin 1929.

H a s se l r o t, Berndt, Något ang. köplagens bestämmelser i $\$ \S 1$ ff. Omtryckt. [nebst] Tillägg. Malmö 1927-28.

H e ck, Phillip, Grundriß des Schuldrechts. Tübingen 1929.

H e Il a u e r, Josef, Kaufverträge, Werk-, Konsignations- und Leihverträge in Warenhandel und Industrie. Berlin 1927. (Bücherei der praktischen Betriebsführung. Bd. 1.)

Hellwig, Konrad, Lehrbuch des deutschen ZivilprozeBrechts. Bd. 1-3, 1 . Leipzig 1903-08.

H e y ma n n, Ernst, Das Verschulden beim Erfüllungsverzug. Zugl. e. Beitrag z. Geschichte d. Obligationenrechts. In: Festgaben für Ludwig Enneccerus dargebracht von den Mitgliedern der Juristischen Fakultät zu Marburg a. L. Marburg 1913.

He ymann-Mosse, HGB.: Mosse, Albert, Handelsgesetzbuch (ohne Seerecht). Neubearb. von Ernst Heymann. 17. Aufl. Berlin \& Leipzig 1926. (Guttentagsche Sammlung deutscher Reichsgesetze.) 
$\mathrm{H}$ of $\mathrm{m}$ a $\mathrm{n} \mathrm{n}, \mathrm{L}$. C., Het Nederlandsch verbintenissenrecht. D. 1. De allgemeene leer der verbintenissen. 2. dr. Groningen 1932. [4. dr. 1935.]

Hu c, Th., Commentaire théorique et pratique du code civil. T. 7. 10. Paris $1894-97$.

Ishizaki, Masaichiro, Le Droit corporatif international de la vente de soies. Vol. 1-3. Paris 1928. (Bibliothèque de l'institut de droit comparé de Lyon. Etudes et documents. T. 18-20.)

$\mathrm{J}$ a e g e r, Ernst, Kommentar zur Konkursordnung und den Einführungsgesetzen, 6. u. 7. Aufl. Bd. 1. 2. Berlin \& Leipzig 1931-36.

Ja eger, Schuldbetreibung: Jaeger, C., Das Bundesgesetz betreffend Schuldbetreibung und Konkurs. 3. vollst. neubearb. Aufl, Bd. 1-3 [nebst] Erg. 1-4. Zürich 1911-34.

Je nks, Edward, A Digest of English civil law. 2. ed. Vol. 1. 2. London 1921.

J o n e s, Leonhard A., The Law of chattel mortgages and conditional sales. $G$. ed. rev. and enlarged by Renzo D. B owers. Vol. 1-3. Indianapolis 1933.

J ossera nd, Louis, Cours de droit civil positif français. 2. éd. T. 1-3. Paris $1932-33$.

Josserand, Louis, Les Transports en service intérieur et en service international. (Transports ferroviaires, roulages, navigation intérieure et navigation aérienne.) A l'exclusion des transports maritimes. 2. éd. Paris 1926.

Juris-Clas seur Civil. Art. 1-2281 [nebst] annexes 1-3. Paris $1913 \mathrm{ff}$. (Collection des Juris-Classeurs.)

K I a n g: Kommentar zum Allgemeinen Bürgerlichen Gesetzbuch. Hrsg. von Heinrich Klang. Bearb. v. Bettelh eim, Pisko [u. a.] Bd. 1-4. Wien 1927-35.

K r u s e, Fr. Vinding, Ejendomsretten. D. 1-5. København 1929-33.

L a nd IV: Land, N. K. F., Verklaring van het burgerlijk wetboek. D. 4. 2. dr. Herzien door Ihr. W. H. de Savornin L oh ma n n. Haarlem 1907.

L a nd V: Land, N. K. F., Verklaring van het burgerlijk wetboek. D. 5. 2. dr. Herzien door C. W. Star B u s m a n n. Haarlem 1915.

$\mathrm{L}$ a r o $\mathrm{mb}$ i è r e, L., Traité théorique et pratique des obligations, ou commentaire des titres 3 et 4 , livre 3, du Code civil art. 1101 à 1386 . Vol. 1-7. Nouv. éd. Paris 1885.

L a s se n, Jul., Haandbog i Obligationsretten. Almindelig Del. 3. tildels omarb. Udg. København 1917-20.

L a s s e $\mathrm{n}-\mathrm{U}$ s s ing: Lassen, Jul., og Henry Ussing, Haandbog i Obligationsretten. Speciel Del. 1. Gave Køb og Bytte. København 1923.

La urent, F., Principes de droit civil français. 3. éd. T. 1-33 [nebst] suppl. T. 1-8. Bruxelles 1878-1903.

L e a k e, S. Martin, Principles of the law of contracts, 8. ed. by R. R. A. W a 1$\mathrm{k}$ e r. London 1931.

Le o, M., Die neuen Geschäftsbeđingungen des deutschen Ausfuhrhandels. Mannheim, Berlin, Leipzig 1929. (Überseestudien z. Handels-, Schiffahrts- u. Versicherungsrecht. $H, 10$.

Le on h a rd, Franz, Das Schuldrecht des BGB. Bd. 1: Allgemeines Schuldrecht des BGB. Bd. 2: Besonderes Schuldrecht des BGB. München \& Leipzig 1929-1931. (Systematisches Handbuch der Deutschen Rechtswissenschaft. Abt. 10. T. 2. Bd. 1. 2.)

L e on h a rd, Franz, Die Beweislast. 2. Aufl. Berlin 1926.

L l e r e na, Baldomero, Concordancias y comentarios del código civil argentino. 3. ed. T. 1-10. Buenos Aires 1931.

Llew elly n, Karl N., Cases and materials on the law of sales. Chicago 1930.

L o m o n a co, Giovanni, Delle obbligazioni e dei contratti in genere. 2. ed. d. F. De g n i. Vol. 1-3. Napoli 1912-15. (Il diritto civile italiano secondo la dottrina e la giurisprudenza. P. 10.)

L y on - Ca ë n, Ch., et L. Rena ult, Traité de droit commercial. 5. éd. T. 1-7. Paris 1921-34.

M a chado, José Olegario, Exposición y comentario del código civil argentino. T. 1-11. Buenos Aires 1922. 
M a la ga r riga, Carlos C., Código de comercio comentado según la doctrina y la jurisprudencia. 2. ed. T. 1-9. Buenos Aires 1922-25. (Derecho comercial argentino)

Manresa y Navarro, José Maria, Comentarios al código civil español. T. 8. 3. ed. Madrid 1918; T. 10. 4. ed. Madrid 1931.

Mariash, Irving, A Treatise on the law of sales under the common law, Uniform Sales Act and other uniform legislation. Albany 1930.

M a r, Robert, Lehrbuch des bürgerlichen Rechtes. Bd. 1. 2. Reichenberg $1922-23$.

Mentzel, Franz, Kommentar zur Reichskonkursordnung. 4. neubearb. Aufl., Mannheim, Berlin, Leipzig 1932.

Mole $n g$ ra a f $f$, W. L. P. A., Leidraad bij de beoefening van het Nederlandsche handelsrecht. 6. dr. D. 1. 2. Haarlem 1930-33.

Mos $\mathrm{e}-\mathrm{H}$ e y m a n $\mathrm{n}$ : $\mathrm{s}$. Heymann-Mosse.

Müller-Erzbach, Rudolf, Deutsches Handelsrecht. 2. u. 3. Aufl. Tübingen 1928.

Mülle re is e r t, F. A., Allgemeine Lieferungsbedingungen der Industrie, des Handwerks und der öffentlichen Hand. Berlin 1932.

$\mathrm{N}$ a va r rin i, Umberto, Trattato teorico-pratico di diritto commerciale. Vol. 2-6; vol. 1. 2. ed. Torino 1920-31.

Oe rtma n n, Paul, Bürgerliches Gesetzbuch. Buch 1. Allg. Teil. 3. umgearb. Aufl. Berlin 1927. Buch 2. Recht d. Schuldverhältnisse. Abt. 1. 2. 5. umgearb. Aufl. Berlin 1928-29. (Kommentar z. Bürgerlichen Gesetzbuch u. seinen Nebengesetzen.)

Oertman n, Paul, Die Geschäftsgrundlage. Ein neuer Rechtsbegriff. Leipzig 1921.

O s e r, Hugo, unter Mitw, von Wilhelm Sch ö n e n be r ge r, Das Obligationenrecht. Bundesgesetz betrefiend die Ergänzung des schweizerischen Zivilgesetzbuchs vom 30. März 1911. 2. umgearb. Aufl. Zürich 1929. (Kommentar zum schweizerischen Zivilgesetzbuch hrsg. von A. Egger, Arnold Escher [u. a.] Bd. 5.)

P a c ch i on i, Giovanni, Corso di diritto civile. Dei contratti in generale. Torino 1933.

Pacifici-Mazzoni, Emidio, Istituzioni di diritto civile italiano. 5. ed. a cura di Giulio Venzi. Vol. 1-7. Firenze 1920-26.

Pac ifici-Mazzoni (Venzi): Pacifici-Mazzoni, Emidio, Il codice civile italiano commentato con la legge romana, le sentenze dei dottori e la giurisprudenza. 6.-8. ed. riv. dal Giulio Venzi. Vol. 1-16. Torino 1927-30.

Pag e, William Herbert, The Law of contracts. 2. ed. rev., rewritten and enlarg. with forms. Vol. 1-7 [nebst] Suppl. 1. 2. Cincinnati 1920-29.

Pa la g i, Dario, La Vente en droit égyptien mixte. Alexandrie 1932.

Percerou, J., Des Faillites et banqueroutes et des liquidations judiciaires. 2. éd. T. 1. Paris 1935. (Ancienne collection Thaller continuée sous la direction de M. J. Percerou.)

P i s k o, Oskar, Lehrbuch des österreichischen Handelsrechtes. Wien 1923.

Plancks Kommentar zum Bürgerlichen Gesetzbuch nebst Einführungsgesetz. Hrsg. von E. Strohal. 4., völlig neubearb. Aufl. Bd. 1. Allg. Teil; Bd. 2. Recht d. Schuldverhältnisse. Berlin 1913-14.

Pla niol, Marcel, Traité élémentaire de droit civil conforme au programme officiel des facultés de droit. 10. éd. avec la collab. de Georges R i pe r t T. 1-3. Paris 1925-27.

Pla niol, Marcel, et Georges $\mathrm{Ripert}$, Traité pratique de droit civil français. T. 6. Obligations. P. 1. Avec le concours de Paul Es me in. Paris 1930. T. 7. Obligations. P. 2. Avec le concours de Paul Es m e in, Jean Ra d oua n t,... Paris 1931.

T. 10. Contrats civils. P. 1. Avec le concours de Joseph $\mathrm{Ham}$ el.. Paris 1932.

T. 12. Sâretés réelles. P. 1. Avec le concours de Emile B e c qué. Paris 1927. 
Pol a c co, Vittorio, Le obbligazioni nel diritto civile italiano. 2. ed. riv. ed ampl. Vol. 1. Roma 1915. (Collezione di opere giuridiche ed economiche.)

Polak: Veegens, J. D., u. A. S. Oppenheim, Schets van het Nederlandsch burgerlijk recht. D. 3. Verbintenissen, bewijs en verjaring. 4. dr. bewerkt door C. H. F. Polak. Haarlem 1934.

Pollo ck, Frederick, The Law of torts. 13. ed. London 1929.

P oll o ck, Frederick, Principles of contract. 9. ed. London 1921.

Pothier, Oeuvres annotées et mises en corrélation avec le Code civil et la législation actuelle par M. Bugnet. T. 2. Obligations. T. 3. Traité du contrat de vente. Traité des retraits. Traité du contrat de constitution de rente. Paris $1847-48$.

Puchelt, Ernst Sigismund, Commentar zum Allgemeinen Deutschen Handelsgesetzbuch. 2. verm. u. verb. Aufl. Bd. 1. 2. Leipzig 1876.

R a b e l, Ernst, Die Haftung des Verkäufers wegen Mangels im Rechte. T. 1. Geschichtliche Studien über den Haftungserfolg. Leipzig 1902.

R a me 11 a, Agostino, La vendita nel moderno diritto. Vol. 1. 2. Milano 1920.

Ra mella, Agostino, Trattato del fallimento. 2. ed. riv. ed ampl. Vol. 1. 2. Milano 1915.

Répertoire du Droit Belge: Répertoire pratique du droit belge. Législation, doctrine et jurisprudence, publié sous la direction de Emile Brunet, Jean Servais [u. a.] T. 3. Contrat et convention - divorce et séparation de corps. Bruxelles 1931.

Répertoire général alphabétique du droit français. Publié sous la dir. de Ed. F u z i e r-H e rma n. Vol. 1-37. [nebst] suppl. 1-13. Paris 1882-1935.

Restatement of the law of contracts as adopted and promulgated by the American Law Institute. Vol. 1. 2. St. Paul 1932.

RGRKomm.: Das Bürgerliche Gesetzbuch mit besonderer Berücksichtigung der Rechtsprechung des Reichsgerichts erl. von Bessau, Hallamik [u. a.] 8. wes. umgearb. Aufl. Bd. 1-5. Berlin \& Leipzig 1934-35.

Rheinstein, Max, Die Struktur des vertraglichen Schuldverhältnisses im anglo-amerikanischen Recht. Berlin \& Leipzig 1932. (Beiträge z. ausländ. u. internat. Privatrecht. H. 5.)

Ricci, Francesco, Corso teorico-pratico di diritto civile. 3. ed. riv. e corr. Nuova ristampa con app. di legislazione. Vol. 1-10. Torino 1923.

Ripert, Georges, Droit maritime. 3. éd. T. 1-3. Paris 1929-30.

Rosenberg. Leo, Die Beweislast auf der Grundlage des Bürgerlichen Gesetzbuchs und der Zivilprozeßordnung. 2. völlig neubearb. Aufl. Berlin 1923.

Rue h l, Helmut, Eigentumsvorbehalt und Abzahlungsgeschäft. Einschl. d. Rechts d. Teilzahlungsfinanzierung. Berlin 1930. (Abhandlungen aus d. Berliner Jurist. Fakultät. 6.)

Ru g g i e r o, Roberto de, Istituzioni di diritto civile. 6. ed. riv, e ampl. Vol. 1-3. Messina 1932-33. [7. ed. riv. 1934-35.]

Salmond, John, and Percy H. W in field, Principles of the law of contracts. London 1927.

Schirrme ister, Gustav u. Wilhelm Proch ownick, Das bürgerliche Recht Englands. Hrsg. von der Internat. Vereinigung f. vergl. Rechtswissenschaft u. Volkswirtschaftslehre zu Berlin. Kodifikation von Edward Jenks, W. M. Geldart [u. a.] Bd. 1. 2. [nebst] Nachtr. Berlin 1906-29.

$\mathrm{Sch}$ wob, Georges, Les Contrats de la London Corn Trade Association (Vente CAF). Paris 1928.

Sedg wick, Theodore, A Treatise on the measure of damages. 9. ed. rev., rearrang., and enlarged by Arthur G. Sedgwick and Joseph H. Beale. Vol. 1-4. New York 1912.

Siber, Heinrich: GrundriB des deutschen bürgerlichen Rechts. 2. Schuldrecht. Leipzig 1931. 
Smith-Gutteridge (Goitein): Smith, John William, A Compendium of mercantile law. 13. ed. by H. C. Gutteridge, H. Goitein, and H. J. S. Jenkins. London 1931.

St a n g, Norsk Formueret: Stang, Frederik, Inledning til Formueretten. 3. Udg. Osio 1935. (Norsk Formueret.)

$S$ t a u b Sommentar zum Handelsgesetzbuch. 14. Aufl. bearb. von Albert P inner, Felix B ondi, Wilhelm Gadow, Eduard Heinichen. Bd. 1-4. Berlin \& Leipzig 1932-33.

$\mathrm{S} t \mathrm{a}$ u b - P i s k o: Staub, Hermann, Kommentar zum Allgemeinen Deutschen Handelsgesetzbuch. Ausg. i. Osterreich bearb. von Oskar Pisko. 3. Aufl. Bd. 1. T. 1. Bd. 2. Lfg. 1-5. Wien 1933-35. Im übrigen 2. Aufl. Wien 1908-10.

Stauding er, J. v., Kommentar zum Bürgerlichen Gesetzbuch und dem Einführungsgesetze. 9. neubearb. Aufl. Bd. 1. Allg. Teil. Erl. von Theodor Loewenfeld und Erwin Riezler. Berlin, München, Leipzig 1925. Bd. 2. Recht d. Schuldverhältnisse. Erl. von Alfred W e r n e r, Karl Kober, [u. a.] T. 1-3. München, Berlin, Leipzig 1925-30.

St olf i, Nicola, Diritto civile. Vol. 1, Parte generale. Vol. 3, Le obbligazioni in generale. Vol. 4, I contratti speciali. Torino 1919-34.

Stulz, Günter, Der Eigentumsvorbehalt im in- und ausländischen Recht. 3. erw. Aufl. Hrsg. von Reichsverband d. deutschen Industrie. Berlin 1932.

Suijling, J. Ph., Inleiding tot het burgerlijk recht. 2. dr. stuk 2. ged. 1. Haarlem 1934. stuk 2. ged. 2. Haarlem 1925.

T a r t u f a r i, Luigi, Della vendita e del riporto. 5. ed. riv. Torino 1925. (II Codice di commercio commentato Vol. 3.) [6. ed. riv. ed. aum. a cura del Enrico Soprano. Torino 1936.]

Thaller, E., Traité élémentaire de droit commercial à l'exclusion du droit maritime. 8. éd. par J. Percerou. T. 1. 2. Paris 1931.

Titze, Heinrich, Bürgerliches Recht. Recht der Schuldverhältnisse. 4. erw. Auf. Berlin 1932. (Enzyklopädie d. Rechts- u. Staatswissenschaft. Abt. Rechtswiss. 8.)

Titze, Heinrich, Die Unmöglichkeit der Leistung nach deutschem bürgerlichen Recht. Leipzig 1900.

Troplong, R., Le Droit civil expliqué suivant l'ordre des articles du code. De la vente. 2. éd. T. 1. 2. Paris 1836 .

T u h r, Andreas von, Allgemeiner Teil des schweizerischen Obligationenrechts. Halbbd. 1. 2. Tübingen 1924-25.

Un dé n, Östen, Svensk sakrätt. 1. Lös egendom. Lund 1927.

Us sing, Henry, Aftaler paa Formuerettens omraade. København 1931.

V e le z, Fernando, Estudio sobre el derecho civil colombiano. 2. ed. corr. y aum. por el autor y por Luis-Angel Arango. 1-9. Paris 1926.

V e nzi, Giulio, Manuale di diritto civile italiano. 7. ed. Torino 1933.

Viva nte, Cesare, Trattato di diritto commerciale, 5. ed. riv, e ampl. Vol. 1-4. Milano 1922-26.

Wijnveldt, J., Burgerlijk wetboek. Rechtspraak, literatuur en korte aanteekeningen. Ged. 3. B. 3. stuk 1. Art. 1269-1348. 4. dr. 's-Gravenhage 1934. (Léon's Rechtspraak. D. 2. afl. 3.)

Willia m' Law and practice in bankruptcy. 14. ed. by Wintringham Norton St a b le and John Basil B la g de n. London 1932.

Will iston: Williston, Samuel, The Law governing sales of goods at common law and under the Uniform Sales Act. 2. ed. Vol. 1. 2. New York 1924.

Willist on, Contracts: Williston, Samuel, The Law of contracts. Vol. 1-5. New York 1922-24.

Winds cheid, Bernhard, Lehrbuch des Pandektenrechts. 9. Aufl, unter vgl. Darst. d. deutsch. bürgerl. Rechts bearb. von Theodor $\mathrm{Kipp}$. Bd. 1-3. Frankfurt a. M. 1906.

Wo od w a rd, Frederic Campbell, The Law of quasi contracts. Boston 1913. 
Gesetze, Entscheidungssammlungen und Zeitschriften sind so, wie in den einzelnen Ländern üblich, zïtiert.

Für Deutschland vgl. G. Maas u. J. Magnus, Abkürzungsverzeichnis der Rechtssprache. Abkürzungen d. deutsch. u. österreich. Rechts. Berlin und Leipzig 1929,

für Österreich vgl. die Angaben bei Klang, Bd. I, 1 S. 9,

für Frankreich vgl. die Nachweise in der Gazette du Palais,

für Italien vgl. unsere Z. 3, 274 N. 1, ferner die Angaben in den Repertorien des Foro Italiano und der Giurisprudenza Italiana,

für Skandinavien Almén I p. XXXVIII ss.

für England vgl. die Angaben in Zivilgesetze der Gegenwart, begründet von K. Heinsheimer. Bd. 2. Das Zivilrecht Englands 1. Teil, Mannheim 1931. S. 79 ff. und

für das gesamte anglo-amerikanische Recht Bouvier's Law Dictionary, Baldwin's century ed. 1934. Ed. by W. E. Baldwin [nebst] suppl. Cleveland 1934. vo. Abbreviation.

In den spanisch-portugiesischen Ländern, in denen keine einheitliche Ubung besteht, sind hauptsächlich folgende Abkürzungen verwendet:

Spanien: z. B. T. S. 30. 10. 1930, Sent. 196, $519=$ Entscheidung des Tribunal Supremo v. 30. 10. 1930, veröffentlicht in Revista general de legislación y jurisprudencia. (Fundada por el José Reus y Garcia Angel Ossario y Gallardo. 3. Jurisprudencia civil. T. 1-217. Madrid 1853-1935) Bd. 196 S. 519.

Argentinien: z. B. Cám. com. de la cap. oder Cám. civ. 2 a de la cap. 22. 11. 1926, Jur. Arg. 23, $438=$ Entscheidung der 2. Zivilkammer oder der Handelskammer der Hauptstadt v. 22. 11. 1926, veröffentlicht in Jurisprudencia Argentina. (T. 1-38 [nebst] Repertorio en los T. 1-36. Buenos Aires 1918-33) Bd. 23 S. 438.

Die Artikel des Código civil nach alter Zählung, welche das Ehegesetz nicht mitrechnet, werden den Artikelnummern neuer Zählung in Klammern beigefügt.

Brasilien: z.B. App. Rio de J. 3. 7. 1925, Rev. de Dir. 80, $579=$ Entscheidung des Appellhofs Rio de Janeiro v. 3. 7. 1925 veröffentlicht in Revista de direito civil, commercial e criminal. (Fundada pelo Antonio Bento de Faria. Vol. 1-115. [nebst] ind. d. vols. 1-60. Rio de Janeiro 1906-35) Bd. 80 S. 579.

Chile: z. B. S. C. 16. 3. 1929 Rev. de Der. 27 II 1, $190=$ Entscheidung der Corte Suprema v. 16. 3. 1929 veröffentlicht in Revista de derecho y jurisprudencia ( $7 \mathrm{ff}$.: Revista de derecho, jurisprudencia y ciencias sociales) Año 1-30. Santiago de Chile 1905-33, Bd. 27, Teil 2, 1. Abteilung S. 190 . 